\title{
EM-Based Maximum-Likelihood Sequence Detection for MIMO Optical Wireless Systems
}

\author{
Nestor D. Chatzidiamantis*, Murat Uysal ${ }^{\dagger}$, Theodoros A. Tsiftsis*, and George K. Karagiannidis* \\ * Department of Electrical \& Computer Engineering, Aristotle University of Thessaloniki, Thessaloniki, Greece, Emails: \{nestoras, thtsif, geokarag\}@auth.gr \\ ${ }^{\dagger}$ Department of Electrical \& Computer Engineering, University of Waterloo, Waterloo, Ontario, Canada, Email: muysal@ece.waterloo.ca
}

\begin{abstract}
A major performance-limiting factor in terrestrial optical wireless (OW) systems is turbulence-induced fading. Exploiting the additional degrees of freedom in the spatial dimension, multiple laser transmitters combined with multiple receiver apertures provide an effective solution for fading mitigation. Although MIMO (Multiple-Input Multiple-Output) OW systems have been extensively studied in recent years, most of these works are mainly limited to symbol-by-symbol decoding. Maximum Likelihood Sequence Detection (MLSD) exploits the temporal correlation of turbulence-induced fading and promises further performance gains. In this paper, we investigate MLSD for IM/DD (intensity-modulation/direct-detection) MIMO OW systems over log-normal atmospheric turbulence channels. Even with a low-order modulation scheme such as On-Off keying which is typically used in OW systems, the complexity of MLSD might be prohibitive. We therefore present an iterative sequence detector based on the expectation-maximization (EM) algorithm. The complexity of the proposed algorithm is much smaller than a direct evaluation of the log-likelihood function. The Monte-Carlo simulation results demonstrate that the EMbased algorithm outperforms the symbol-by-symbol decoder and achieves a performance which lies within $0.5 \mathrm{~dB}$ of that of the optimal MLSD.
\end{abstract}

\section{INTRODUCTION}

Wireless technology is traditionally associated with radiofrequency $(\mathrm{RF})$ transmission although transmission via carriers in the other parts of electromagnetic spectrum might be more advantageous for various applications [1]. Operating at unlicensed optical wavelengths, terrestrial optical wireless (OW) systems offer the potential of broadband communication capacity. The major limitation in the performance of $\mathrm{OW}$ systems is their high vulnerability to adverse atmospheric conditions. Even in a clear sky, due to inhomogeneities in temperature and pressure changes, the refractive index of the atmosphere varies and results in atmospheric turbulence. This causes rapid fluctuations at the received signal, known as turbulence-induced fading. Such fluctuations lead to an increase in the error rate performance, thereby severely affecting the reliability of OW link.

Over the last years, several fading mitigation techniques have been proposed for deployment in OW links to combat the degrading effects of atmospheric turbulence. One of such techniques is error control coding (ECC) which has been investigated in [2] and [3]. ECC in conjunction with interleaving is known in the RF literature to provide an effective timediversity solution for rapidly-varying fading channels. In the OW systems, data rates can typically be of the order of gigabits per second. With a correlation time of the order of $10^{-3}$ to $10^{-2}$ seconds, OW channels exhibit slow fading; therefore the practical use of ECC in OW links is rather limited due to the required large-size interleavers to achieve the promising coding gains theoretically available.

An effective solution for fading mitigation is spatial diversity techniques which involve the deployment of multiple transmit/receive apertures. Introducing additional degrees of freedom in the spatial dimension, MIMO (multiple-input multiple-output) OW systems promise significant performance gains. The performance of MIMO OW systems has been extensively studied by various authors assuming different type of fading channels, noise statistics and modulation type [4][8]. These works however are mainly limited to the assumption of symbol-by-symbol decoding. It is known that Maximum Likelihood Sequence Detection (MLSD) exploits the temporal characteristics of the fading channels and outperforms the conventional symbol-by-symbol maximum likelihood (ML) decoder. It proves to be particularly effective for slowly-varying fading or quasi-static fading channels as the turbulenceinduced fading turns out to be. MLSD in the context of OW communication has been investigated by Zhu and Kahn in [9], [10] and [11] for a SISO (single-input single-output) scenario. However, MLSD involves the computation of complicated multidimensional integrals and therefore suffers from high complexity. To address the complexity issue, sub-optimal, yet of low-complexity, MLSD algorithms have been proposed in [10]. Other detection methods such as pilot-symbol assisted modulation (PSAM) and multi-symbol detection have been also applied to SISO OW systems in [12]- [14].

In this work, we aim to investigate MLSD solutions for MIMO OW systems as a powerful combination for fading mitigation in atmospheric turbulence channels. Since MLSD in a MIMO scenario suffers from excessive complexity and is infeasible for most practical purposes, we propose an iterative ML sequence detector based on the expectation-maximization (EM) algorithm. The EM algorithm has been originally proposed by Dempster, Laird, and Rubin [15] in the statistics literature as a general procedure for iterative ML estimation. Since then, it has been widely applied to a variety of communication problems [16]- [18]. This algorithm is particularly useful when the estimation problem is made difficult by the absence of certain information, e.g., absence of full channel state information in a data detection problem. In this paper, we 
propose EM algorithm as a low-complexity solution for MLSD in the MIMO OW system under consideration. As benchmarks, we also investigate the performance of symbol-by-symbol ML detector, PSAM and MLSD in a MIMO scenario. The proposed detector outperforms both symbol-by-symbol ML detector and PSAM methods. Moreover, its performance lies within $0.5 \mathrm{~dB}$ of the MLSD at a much lower complexity.

The remainder of the paper is organized as follows. In section II, we describe the MIMO OW system and the turbulenceinduced fading model. In section III, we present symbolby-symbol and sequence-based ML detectors, which will be used as benchmarks for system performance and complexity, and introduce the EM algorithm for the MIMO OW system under consideration. In section IV, we present Monte-Carlo simulation results to demonstrate the error rate performance of the proposed detection technique and compare it with benchmarking schemes. Finally, in section V, we provide our concluding remarks.

Notations: $(\boldsymbol{x})^{T}$ denotes the transpose of the matrix $\boldsymbol{x}$; $E[\cdot]$, denotes statistical expectation; $\|\boldsymbol{x}\|$ denotes the norm of the vector $\boldsymbol{x} ; N\left(\mu, \sigma^{2}\right)$ denotes Gaussian distribution with mean $\mu$ and variance $\sigma^{2}$.

\section{System And Channel Model}

We consider a MIMO OW link with $N$ transmit and $M$ receive apertures. At the transmitter, data blocks of length $L$ are modulated using On-Off keying (OOK) and transmitted through the $N$ apertures using repetition coding [19].

We assume operation in the high signal-to-noise ratio (SNR) regime where the shot noise caused by ambient light is dominant and therefore Gaussian noise model is used as a good approximation of the Poisson photon counting detection model [9]. Furthermore, a large field of view is considered for each receiver indicating that multiple transmitters are simultaneously observed by each receiver. This actually leads to the collection of larger amount of background radiation which further justifies the use of Gaussian noise model.

Let $s=\left(\begin{array}{cccc}s^{(1)} & s^{(2)} & \ldots & s^{(L)}\end{array}\right)^{T}$ be the $L \times 1$ column vector containing the $L$ modulated symbols within a data frame. The received signal at the $m$ th receive aperture is given as

$$
r_{m}^{(l)}=s^{(l)} \eta \sum_{n=1}^{N} I_{n m}^{(l)}+v_{m}, m=1, \ldots, M, l=1, \ldots L
$$

where $s^{(l)} \in\{0,1\}$ is the transmitted information bit, $\eta$ is the optical-to-electrical conversion coefficient, and $v_{m}$ is additive white Gaussian noise (AWGN) with zero mean and variance $\sigma_{v}^{2}=N_{o} / 2$. The fading coefficient, which models the atmospheric turbulence in the optical channel between the $n$th transmit aperture and the $m$ th receive aperture during the $l$-th symbol interval, is given by

$$
I_{n m}^{(l)}=I_{o} \exp \left(2 x_{n m}^{(l)}\right)
$$

where $I_{o}$ is the signal light intensity without turbulence and $x_{n m}^{(l)}$ are identically distributed normal random variables with mean $\mu_{x}$ and variance $\sigma_{x}^{2}$, i.e. $f_{x}\left(x_{n m}^{(l)}\right)=N\left(\mu_{x}, \sigma_{x}^{2}\right)$. Therefore, $I_{n m}^{(l)}$ follows a lognormal distribution with probability density function (pdf) given by

$$
f\left(I_{n m}^{(l)}\right)=\frac{1}{2 I_{n m}^{(l)}} \frac{1}{\sqrt{2 \pi \sigma_{x}^{2}}} \exp \left(-\frac{\left(\ln \left(\frac{I_{n m}^{(l)}}{I_{o}}\right)-2 \mu_{x}\right)}{8 \sigma_{x}^{2}}\right)
$$

To ensure that the fading does not attenuate or amplify the average power, we normalize the fading coefficients such that $\left.E\left[\mid \frac{I_{n m}^{(l)}}{I_{o}}\right]\right]=1$. Doing so requires the choice of $\mu_{x}=-\sigma_{x}^{2}$ [8]. The variances of log-amplitude fluctuations of plane and spherical waves can be found in [20].

Atmospheric turbulence results in a very slowly-varying fading in OW systems. For the signalling rates of interest ranging from hundreds to thousands of Mbps [21], the fading coefficient can be considered as constant over hundred of thousand or millions of consecutive symbols, since the coherence time of the channel is about 1-100ms [4]. Hence, it is assumed that $I_{n m}^{(l)}$ remains constant over the symbols of a frame and therefore we drop the time index $l$, i.e.

$$
I_{n m}^{(l)}=I_{n m}, l=1, . . L .
$$

Moreover, it is assumed that the distances between the transmitter and receiver apertures are large compared to the correlation length of intensity fluctuations. Hence, the underlying channels can be considered as independent in space.

At the receiver, we assume equal gain combining (EGC). Thus, after combining the received signals of the $M$ apertures, the output of the receiver can be written in matrix form as

$$
\boldsymbol{r}=\frac{1}{N M} \sum_{m=1}^{M} \boldsymbol{r}_{m}=\frac{\eta I_{o}}{N M} \boldsymbol{s} \sum_{m=1}^{M} \sum_{n=1}^{N} I_{n m}+\boldsymbol{n}
$$

where $\boldsymbol{n}$ is a $L \times 1$ column vector which contains noise samples. Note that a scaling factor $N M$ appears in (5). The factor $N$ is included in order to ensure that the total transmit power is the same with that of a system with no transmit diversity. The factor $M$, on the other hand, ensures that the sum of the $M$ receive aperture areas is the same with the aperture area of a system with no receive diversity.

\section{ML Detection TechniQues for MiMO OW SYSTEMS}

In this section, we present detection techniques which are based on the maximum likelihood (ML) criterion and can be employed when instantaneous channel state information (CSI) is not available at the receiver. It is assumed that the receiver has knowledge of the marginal joint distribution of the intensity fluctuations, but not of their instantaneous state.

\section{A. ML Symbol-by-Symbol Detection}

The ML symbol-by-symbol detector chooses the symbol $\hat{s}$ based on the rule [22]

$$
\hat{s}=\arg \max _{s} p(r \mid s)
$$


where $p(r \mid s)$ is the conditional probability of the received signal $r$ when $s$ is transmitted. The likelihood function for SISO OW systems is given by [9, Eq. 30]. For the MIMO OW system under consideration, the ML decision rule takes the form of

$$
\begin{aligned}
\hat{s}= & \arg \max _{s} \int_{\boldsymbol{x}} f_{\boldsymbol{x}}(\boldsymbol{x}) \\
& \times \exp \left(-\frac{\left(r-\frac{\eta I_{o}}{N M} \sum_{m=1}^{M} \sum_{n=1}^{N} e^{2 x_{n m}}\right)^{2}-r^{2}}{N_{o}}\right) d \boldsymbol{x}
\end{aligned}
$$

where $\boldsymbol{x}=\left\{x_{n m}\right\}$ and $f_{\boldsymbol{x}}(\boldsymbol{x})=\prod_{m=1}^{M} \prod_{n=1}^{N} f_{x}\left(x_{n m}\right)$.

The efficient computation of (7) can be made using the sum of log-normal approximation and Gauss-Hermite quadrature formula as discussed in [8].

\section{B. ML Sequence Detection (MLSD)}

This detection scheme exploits the temporal correlation of turbulence-induced fading over consecutive transmitted symbols. MLSD was first investigated in OW communications [9], assuming a SISO scenario. For the MIMO OW system with OOK, the MLSD computes the likelihood ratio of each of the $2^{L}$ possible sequences and chooses according to the decision rule

$$
\begin{aligned}
\hat{\boldsymbol{s}} & =\arg \max _{\boldsymbol{s}} p(\boldsymbol{r} \mid \boldsymbol{s}) \\
& =\arg \max _{\boldsymbol{s}} \int_{\boldsymbol{x}} f_{\boldsymbol{x}}(\boldsymbol{x}) \\
& \times \exp \left[-\sum_{l=1}^{L} \frac{\left(r^{(l)}-s^{(l)} \frac{\eta I_{o}}{N M} \sum_{m=1}^{M} \sum_{n=1}^{N} e^{2 x_{n m}}\right)^{2}}{N_{o}}\right] d \boldsymbol{x}
\end{aligned}
$$

where $\boldsymbol{r}=\left[r_{1}, r_{2}, \ldots, r_{L}\right]$ represents the received data.

A major drawback of MLSD is its computational complexity, since it requires the computation of the $M N$-dimensional integral of (8) for each of the $2^{L}$ possible sequences. A suboptimal low-complexity implementation has been proposed in [10], adopting Markov model; it still requires the numerical integrations to be performed in each branch metric in the trellis search. In [12]- [13], another efficient low complexity implementation is proposed, which still requires the computation of (8), but for less number of sequences.

\section{EM-Based Sequence Detection}

In this sub-section, we propose an EM-based sequence detector as a low complexity alternative to original MLSD. This scheme is a two-step iterative procedure which estimates both the transmitted sequence and the channel state based on its previous estimates.

With the available data only, i.e., the "incomplete data" set according to the EM terminology [15], finding the ML estimates might be computationally intensive as in our case.
Including a proper selection of another data set resulting in so-called "complete data" set, it might be made easier to compute the ML estimates. Since not all the elements of the complete data set are known, the EM algorithm makes use of the log-likelihood function for the complete data in a two-step iterative procedure, iterating between expectation step (E-step) and maximization step (M-step).

In our work, the received sequence $\boldsymbol{r}$ is the incomplete data set. Let the complete data be $\boldsymbol{y}=\left(\boldsymbol{r},\left\{I_{n m}\right\}\right)$. After using the likelihood function for MIMO OW systems, as in (5), the log-likelihood function for the complete data in vector form, is obtained by

$$
l\left(\boldsymbol{r} \mid s,\left\{I_{n m}\right\}\right)=\frac{1}{N_{o}}\left\|\boldsymbol{r}-\boldsymbol{s} \frac{\eta I_{o}}{N M} \sum_{m=1}^{M} \sum_{n=1}^{N} I_{n m}\right\|^{2}
$$

After dropping some unnecessary terms, (9) reduces to

$$
\begin{aligned}
l\left(\boldsymbol{r} \mid \boldsymbol{s},\left\{I_{n m}\right\}\right)= & \boldsymbol{r}^{T} s \frac{\eta I_{O}}{N M} \sum_{m=1}^{M} \sum_{n=1}^{N} I_{n m} \\
& -\frac{1}{2}\left\|s \frac{\eta I_{O}}{N M} \sum_{m=1}^{M} \sum_{n=1}^{N} I_{n m}\right\|^{2}
\end{aligned}
$$

or equivalently

$$
\begin{aligned}
l\left(\boldsymbol{r} \mid s,\left\{I_{n m}\right\}\right)=\sum_{l=1}^{L} & \left(r^{(l)} s^{(l)} \frac{\eta I_{o}}{N M} \sum_{m=1}^{M} \sum_{n=1}^{N} I_{n m}\right. \\
& \left.-\frac{1}{2}\left\|s^{(l)} \frac{\eta I_{o}}{N M} \sum_{m=1}^{M} \sum_{n=1}^{N} I_{n m}\right\|^{2}\right)
\end{aligned}
$$

At the E-step of the $k$-th iteration of the algorithm, the log-likelihood function for the complete data is calculated, conditioned on the received sequence $r$ and the $k$-th estimate of the transmitted sequence $s^{k}$. Hence, based on (10) or (11) we obtain

$$
Q\left(\boldsymbol{s} \mid \boldsymbol{s}^{k}\right)=\boldsymbol{r}^{T} \mathbf{s} I^{k}-\frac{1}{2}\left\|\boldsymbol{s} I^{k}\right\|^{2}
$$

where

$$
I^{k}=E\left[\frac{\eta I_{o}}{N M} \sum_{m=1}^{M} \sum_{n=1}^{N} I_{n m} \mid s^{k}, \boldsymbol{r}\right]=\frac{1}{L_{o n}^{k}} \boldsymbol{r}^{T} \boldsymbol{s}^{k}
$$

and $L_{o n}^{k}$ is the number of the bits of the $k$-th estimate of the transmitted sequence $s^{k}$ that correspond to the On-state.

A better estimate of the transmitted sequence, $s^{k+1}$, is obtained at the second step of the $k$-th iteration of the algorithm, the M-step. M-step performs the maximization of (12) which yields

$$
\boldsymbol{s}^{k+1}=\arg \max _{\boldsymbol{s}}\left(\boldsymbol{r}^{T} \boldsymbol{s} I^{k}-\frac{1}{2}\left\|\boldsymbol{s} I^{k}\right\|^{2}\right)
$$

Since the transmitted data bits are randomly chosen, i.e. no coding scheme is employed, maximizing (14) over the $L$ bit sequences is equivalent to making symbol-by-symbol decisions on each bit. In other words, if $s^{k+1}$ is the sequence that 


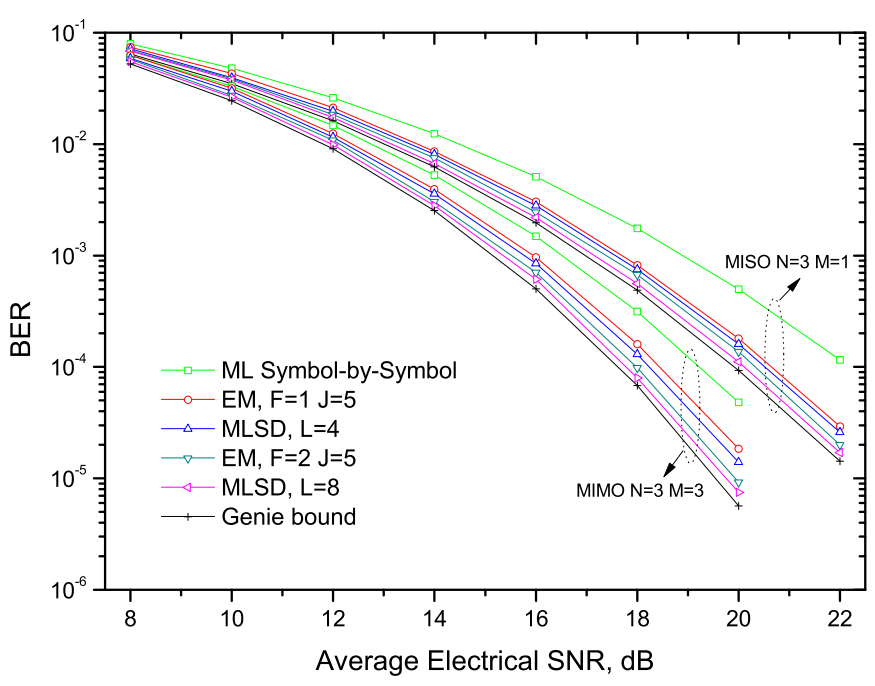

Fig. 1. Comparison of symbol detection techniques for MIMO and MISO OW systems.

maximizes (14), its components are easily obtained through

$$
\left(s^{k+1}\right)^{(l)}=\arg \max _{s^{(l)}}\left(r^{(l)} s^{(l)} I^{k}-\frac{1}{2}\left\|s^{(l)} I^{k}\right\|^{2}\right), l=1, \ldots, L
$$

Unlike MLSD, this detection scheme does not require knowledge of the marginal joint distribution of the intensity fluctuations at the receiver. However, it needs an initial estimate of the channel state, $I^{0}$, in order to initialize the algorithm. Hence, pilot channel estimation bits are inserted at each data frame at the transmitter. Assume that pilot symbols are inserted into the modulated data blocks at every $J$ symbols. In making decisions, the receiver makes observation on data vector of $F$ sub-blocks, each with a length of $J$ (i.e., $L=F \cdot J$ symbols), for some integer $F$. Of the $J$ symbols in the subblock, the first is a pilot symbol, which is in the On-state. The initial estimate of the channel's state is obtained by taking the average of the pilot symbols. The quality of the initial channel estimate and/or the length of the data frames determine the convergence of the EM-based receiver to the perfect CSI receiver [16].

\section{Simulations Results and Discussion}

In this section, we present simulation results for the bit error rate (BER) performance of OW communication system with EM-based receiver for various numbers of transmit/receive apertures. We further compare its performance with other competing detection techniques.

In Fig. 1, we consider a MISO (multiple-transmit singleoutput) OW system with $N=3$ transmit apertures and a MIMO system with $N=3$ transmit and $M=3$ receive apertures over a lognormal turbulence channel with $\sigma_{x}=0.3$. The performance of proposed EM algorithm along with ML symbol-by-symbol decoder and MLSD is illustrated. We assume two iterations in the implementation of EM algorithm since further iterations results in a negligible performance

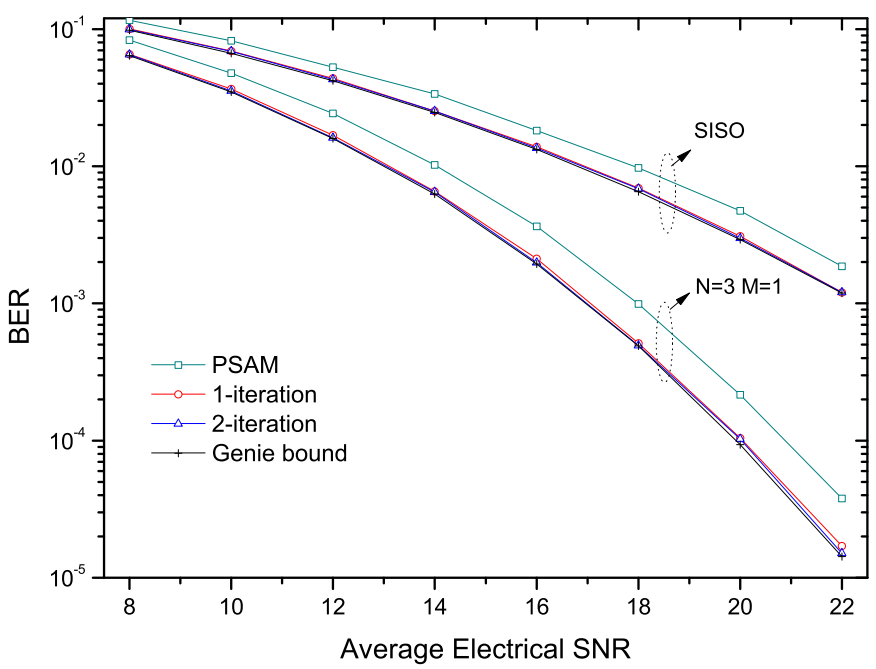

Fig. 2. Performance of EM-based receiver for $F=1$ and $L=100$ and comparison with PSAM.

improvement. Among the three competing schemes, symbolby-symbol detection scheme performs the worst. This is expected, since this detector does not take advantage of the fading correlation between successive symbols that exists in turbulence channels. Hence, the use of sequence detection techniques, such as MLSD or EM is fully justified. As an ultimate benchmark, we also include the performance of genie receiver which assumes perfect CSI and acts as a lower bound on the performance of other detection types. MLSD performs very close to the genie bound and slightly outperforms the EM detector. Specifically, there is an SNR improvement of approximately $0.5 \mathrm{~dB}$ at $\mathrm{BER}=10^{-4}$ between MLSD of $L=4$ and EM of $(F=1, J=5)$ or between MLSD of $L=8$ and EM of $(F=2, J=5)$ in both MIMO OW systems. However, it should be noted that the complexity of the MLSD implementation is much higher than that of EM algorithm, i.e. MLSD requires the calculation of $L \mathrm{NM}$ dimensional intergrals [12]. That is also the same reason why we restricted ourselves to small values of frame lengths. Otherwise, simulation of MLSD would not be possible for comparison purposes.

In Fig. 2, we investigate the performance of EM algorithm for a large frame length, which would be prohibitive for MLSD implementation. Furthermore, large frame lengths make the loss due to pilot insertion negligible. Specifically, we consider the SISO and the MISO OW systems with $L=100$, assuming sub-block length of $J=100$ and pilot symbol number $F=1$. The performance of proposed EM algorithm is illustrated assuming one and two iterations. It is observed that the performance of EM detector lies within $0.1 \mathrm{~dB}$ of the genie bound for $F=1$ and $J=100$. As another benchmark, we also include the performance of PSAM with variable threshold [14], i.e. the decision rule for the detection of each symbol in the frame, is defined entirely by the channel estimate of the pilot symbols. The EM algorithm outperforms PSAM by approximately $1 \mathrm{~dB}$. 


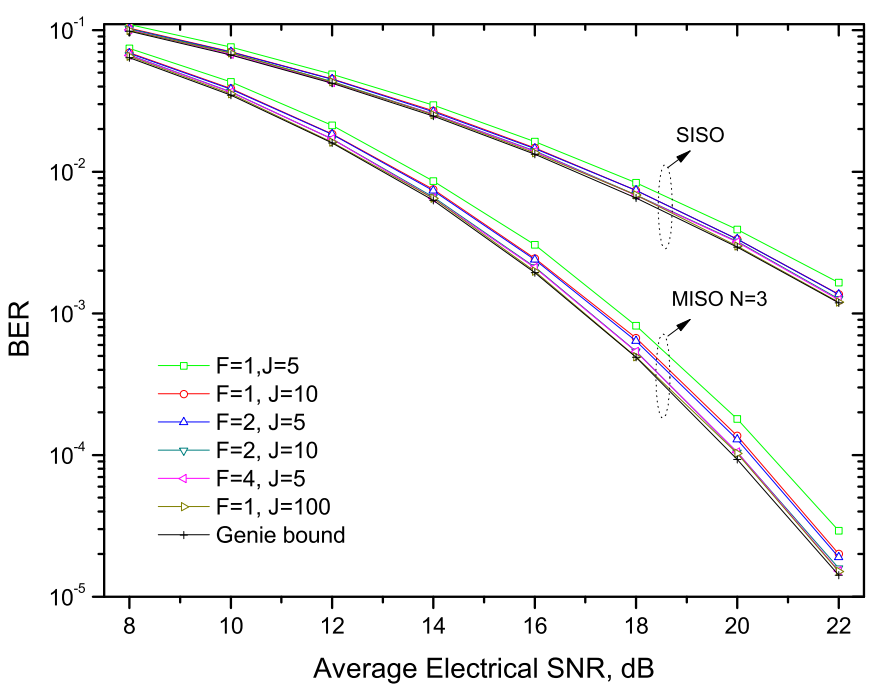

Fig. 3. Performance of EM-based receiver for various number of pilot symbols $F$ and data-block lengths $L(L=F \cdot J)$

Fig. 3 depicts the performance of the EM-based receiver for different frame lengths assuming various combinations of $F$ and $J$ values. Specifically, we assume the combinations of $F=1$ and $J=5, F=1$ and $J=10, F=2$ and $J=5, F=2$ and $J=10, F=4$ and $J=5$ and, finally, $F=1$ and $J=100$. It is observed that the increase of the length and/or the number of pilot symbols helps EM-based receiver to perform closer to the genie bound. It should be noted that, unlike MLSD, the complexity of the algorithm does not increase significantly as the length of data blocks increases. This is expected, since the EM implementation does not involve the computation of $N M$-dimensional integrals and the increase of frame length increases only the number of the symbol-by-symbol decisions performed at the M-step of the algorithm.

\section{CONCLUSIONS}

We have investigated sequence detection techniques for MIMO OW systems in the presence of turbulence-induced fading. Sequence detection exploits the temporal correlation of OW channel and promises significant performance gains over the symbol-by-symbol decoding. However, optimal ML sequence detector is infeasible for most practical purposes due to its high complexity. In our work, we have proposed a lowcomplexity sequence detector based on the EM algorithm. EM algorithm provides an iterative ML solution and its complexity is largely independent of the frame size. Our simulation results demonstrate that the proposed EM solution peforms very close to that of MLSD. Specifically, it has been observed that the performance of EM is merely $0.5 \mathrm{~dB}$ (at $\mathrm{BER}=10^{-4}$ ) away from that of MLSD for OW system under consideration.

\section{REFERENCES}

[1] L. Andrews, R. L. Philips, and C. Y. Hopen, Laser Beam Scintillation with Applications. SPIE Press, 2001.

[2] X. Zhu and J. M. Kahn, "Performance bounds for coded free-space optical communications through atmospheric turbulence channels," IEEE Trans. on Commun., vol. 51, no. 8, pp. 1233-1239, Aug. 2003.

[3] M. Uysal, S. M. Navidpour, and J. Li, "Error rate performance of coded Free-Space optical links over strong turbulence channels," IEEE Communications Letters, vol. 8, no. 10, pp. 635-637, Oct. 2004.

[4] E. Lee and V. Chan, "Part 1: Optical communication over the clear turbulent atmospheric channel using diversity," IEEE Journal on Selected Areas in Commun., vol. 22, no. 9, pp. 71 896-1906, Nov. 2004

[5] S. G. Wilson, M. Brandt-Pearce, C. Qianling, and M. Baedke, "Optical repetition MIMO transmission with multipulse PPM," IEEE Journ. on Sel. Areas in Commun., vol. 23,, no. 9, pp. 1901-1910, 2005.

[6] S. G. Wilson, M. Brandt-Pearce, C. Qianling, and J. Leveque, "FreeSpace Optical MIMO transmission with Q-ary PPM," IEEE Trans. on Commun., vol. 53,, no. 8, pp. 1402-1412, Aug. 2005.

[7] T. A. Tsiftsis, H. G. Sandalidis, G. K. Karagiannidis, and M. Uysal, "Optical wireless links with spatial diversity over strong atmospheric turbulence channels," to appear in IEEE Transactions on Wireless Communications.

[8] S. M. Navidpour and M. Uysal, "BER performance of free-space optical transmission with spatial diversity," IEEE Trans. Wireless Commun., vol. 6, no. 8, pp. 2813-2819, Aug. 2007.

[9] X. Zhu and J. M. Kahn, "Free-space optical communication through atmospheric turbulence channels," IEEE Trans. Commun., vol. 50, no. 8 , pp. 1293-1300, Aug. 2002.

[10] _ "Markov chain model in maximum-likelihood sequence detection for free-space optical communication through atmospheric turbulence channels," IEEE Trans. on Commun., vol. 51, no. 3, pp. 509-516, Mar. 2003.

[11] X. Zhu, J. M. Kahn, and J. Wang, "Mitigation of turbulence-induced scintillation noise in Free-Space Optical links using temporal-domain detection techniques," IEEE Photonics Techn. Lett., vol. 15, no. 4, pp. 623-625, Apr. 2003.

[12] M. L. B. Riediger, R. Schober, and L. Lampe, "Multiple-symbol detection for shot-noise limited free-space optical communications," in CCECE, Vancuver, Canada, 2007, pp. 908-911.

[13] _ - "Reduced-complexity multiple-symbol detection for free-space optical communications," in GLOBECOM, Washington DC, USA, 2007, pp. $4548-4553$.

[14] X. Zhu and J. M. Kahn, "Pilot-symbol assisted modulation for correlated turbulent free-space optical channels," in Proc. of SPIE Intl. Symp. on Optical Science and Technol., San Diego, CA, 2001.

[15] A. Dempster, N. M. Laird, and D. B. Rubin, "Maximum-likelihood from incomplete data via the EM algorithm," J. Roy. Statist. Soc, vol. 39, pp. $1-17,1977$.

[16] C. N. Georghiades and J. C. Han, "Sequence estimation in the presence of random parameters via the EM algorithm," IEEE Trans. on Commun., vol. 45, no. 3, pp. 300-308, Mar. 1997.

[17] C. Cozzo and B. L. Hughes, "Joint channel estimation and data detection in Space-Time communications," IEEE Trans. on Commun., vol. 51, no. 8, pp. 1266-1270, Aug. 2003.

[18] Y. Li, C. N. Georghiades, and G. Huang, "Iterative maximum-likelihood sequence estimation for space-time coded systems," IEEE Trans. on Commun., vol. 49, no. 6, pp. 948-951, Jun. 2001.

[19] M. Safari and M. Uysal, "Do we really need space-time coding for freespace optical communication with direct detection?" to appear in IEEE Trans. Wireless Commun.

[20] S. Karp, R. Gagliardi, S. E. Moran, and L. B. Stotts, Optical Channels. New York: Plenum, 1988.

[21] D. J. T. Heatley, D. R. Wisely, I. Neild, and P. Cochrane, "Optical wireless: the story so far," IEEE Commun. Mag., vol. 36, no. 2, pp. 72-74, Dec. 1998.

[22] J. G. Proakis, Digital Communications, 4th ed. New York: Mc Graw Hill, 2000. 